\title{
Who Thrives Under Pressure? Predicting the Performance of Elite Academy Cricketers Using the Cardiovascular Indicators of Challenge and Threat States
}

\author{
Martin J. Turner, ${ }^{1}$ Marc V. Jones, ${ }^{1}$ David Sheffield, ${ }^{2}$ Matthew J. Slater, ${ }^{1}$ \\ Jamie B. Barker, ${ }^{1}$ and James J. Bell ${ }^{3}$ \\ ${ }^{1}$ Staffordshire University; ${ }^{2}$ University of Derby; ${ }^{3}$ Bangor University
}

\begin{abstract}
This study assessed whether cardiovascular $(\mathrm{CV})$ reactivity patterns indexing challenge and threat states predicted batting performance in elite male county $(N=12)$ and national $(N=30)$ academy cricketers. Participants completed a batting test under pressure, before which $\mathrm{CV}$ reactivity was recorded in response to egothreatening audio instructions. Self-reported self-efficacy, control, achievement goals, and emotions were also assessed. Challenge CV reactivity predicted superior performance in the Batting Test, compared with threat $\mathrm{CV}$ reactivity. The relationships between self-report measures and $\mathrm{CV}$ reactivity, and self-report measures and performance were inconsistent. A small subsample of participants who exhibited threat CV reactivity, but performed well, reported greater self-efficacy than participants who exhibited threat CV reactivity, but performed poorly. Also a small subsample of participants who exhibited challenge reactivity, but performed poorly, had higher avoidance goals than participants with challenge reactivity who performed well. The mechanisms for the observed relationship between $\mathrm{CV}$ reactivity and performance are discussed alongside implications for future research and applied practice.
\end{abstract}

Keywords: emotions, evaluations, cortisol, catecholamines, stress, impedance cardiography

Competition is stressful (Harrison et al., 2001; Salvador, 2005), and for elite athletes, competitive stress is intensified by the career implications of success and failure, and the scrutiny under which they perform (Jordet, 2009). For an elite academy cricketer, performance scrutiny is unremitting even in training, where the athlete is compared with others for team selection under conditions of high expectation, requiring a continuous investment of substantial effort in the pursuit of successful performance. It is within this stressful academy context (Barker, McCarthy, \& Harwood, 2011) that the current study examines whether psychophysiological stress responses predict cricket batting performance under pressure.

In motivated performance situations, such as sport, performance may be disrupted (Seery, 2011). A variety of approaches have described this phenomenon, considering the role of the autonomic nervous system in driving physiological arousal (e.g., catastrophe theory; Hardy,

Martin J. Turner, Marc V. Jones, Matthew J. Slater, and Jamie B. Barker are with the Centre for Sport, Health and Exercise Research, Staffordshire University, Stoke on Trent, U.K. David Sheffield is with the Centre for Psychological Research, University of Derby, Derby, U.K. James J. Bell is with the Institute for the Psychology of Elite Performance, Bangor University, Bangor, Gwynedd, U.K.
1990; multidimensional anxiety theory; Martens, Burton, Vealey, Bump, \& Smith, 1990). However, whether an elite athlete's cardiovascular (CV) reaction to psychological stress can predict performance is yet to be fully understood. In the present paper, CV reactivity to a psychological stressor (i.e., verbal description of a cricket batting test) is used to predict performance in a cricket batting test. That is, individuals' responses to the description of a task are used to predict subsequent performance in the task. The present paper adopts the biopsychosocial (BPS) model of challenge and threat (Blascovich \& Mendes, 2000) as a framework. The BPS model, informed by the work of Lazarus (e.g., Lazarus \& Folkman, 1984) and Dienstbier (1989), proposes two distinct patterns of CV reactivity that distinguish challenge (adaptive response to stress) and threat (maladaptive response to stress). Providing a secondary framework, and based on the BPS model, the theory of challenge and threat states in athletes (TCTSA; Jones, Meijen, McCarthy, \& Sheffield, 2009) brings together psychological and emotional factors that underpin an athlete's performance in motivated performance situations.

At the core of the TCTSA is the notion that some athletes excel in motivated performance situations whereas others fail to perform (Jones et al., 2009). The TCTSA proposes that in competitive situations, demand 
evaluations are made about the extent to which an event prompts perceptions of danger (physical or esteem), uncertainty, and effort (physical and psychological). In the TCTSA, resource evaluations determine whether an individual perceives sufficient or insufficient resources to meet the demands of a situation. The resource evaluations comprise three interrelated constructs: self-efficacy, perceptions of control, and achievement goals. Specifically, high levels of self-efficacy, perceived control, and a focus on approach goals underpin a challenge state, while low levels of self-efficacy, perceived control, and a focus on avoidance goals underpin a threat state. Evidence linking the resource appraisals to challenge and threat states has emerged in a number of studies (e.g., Chalabaev, Major, Cury, \& Sarrazin, 2009; Quested, Bosch, Burns, Cumming, Ntoumanis, \& Duda, 2011).

In the TCTSA, challenge and threat states have their own distinct patterns of CV reactivity, adopted from the BPS model and validated many times empirically (for reviews, see Blascovich, Mendes, Vanman, \& Dickerson, 2011; Seery, 2011). These distinct patterns of CV reactivity are proposed to have differing performance implications. Primarily, the CV indices of challenge and threat are an indicator of an athlete's ability to adapt in motivated performance situations, and therefore may be able to predict performance in competition. A challenge state is accompanied by increased catecholamine output (epinephrine and norepinephrine), indicating sympathetic adreno-medullary activity, which is reflected in increased heart rate (HR) and cardiac output (CO), attenuated preejection period (PEP), and decreased total peripheral resistance (TPR). This challenge $\mathrm{CV}$ reactivity pattern represents an efficient physiological response to stressors, where the energy needed for successful performance (e.g., glucose) is released into the blood and can reach the brain and muscles efficiently due to decreased vascular resistance and enhanced blood flow (Dienstbier, 1989, 1992). Consequently, a challenge state is proposed to facilitate improved decision making, effective and maintained cognitive function, decreased likelihood of reinvestment, efficient self-regulation, and increased anaerobic power-all likely to lead to successful competitive performance (Jones et al., 2009). A threat state is also marked by increased sympathetic adrenomedullary activity, but is accompanied by increased pituitary adreno-cortical activity, accompanied by cortisol release (e.g., Jamieson, Koslov, Nock, \& Mendes, 2013). Thus, increased HR and attenuated PEP occurs, but with an increase or stabilization in TPR, and a small increase, decrease, or stabilization in CO. In this threat $\mathrm{CV}$ reactivity pattern, pituitary adreno-cortical activity is thought to temper sympathetic adreno-medullary activity; therefore, compared with a challenge $\mathrm{CV}$ reactivity pattern, efficient energy delivery to the brain and muscles does not occur (Dienstbier, 1989, 1992). Consequently, a threat state is proposed to lead to ineffective decision making and cognitive function, increased likelihood of reinvestment, inefficient self-regulation, and decreased anaerobic power (compared with a challenge state)—all likely to lead to unsuccessful competitive performance (Jones et al., 2009).

A growing body of research indicates that challenge $\mathrm{CV}$ reactivity can predict superior athletic performance compared with threat (e.g., Blascovich, Seery, Mugridge, Norris, \& Weisbuch, 2004; Moore, Vine, Wilson, \& Freeman, 2012; Turner, Jones, Sheffield, \& Cross, 2012). Blascovich et al. (2004) found that in response to a speech task about competing, athletes who exhibited stronger challenge $\mathrm{CV}$ reactivity performed better over a competitive season, compared with participants exhibiting threat $\mathrm{CV}$ reactivity. Two recent papers build on the work of Blascovich et al., in sport. Moore et al. (2012) found that participants with challenge $\mathrm{CV}$ reactivity performed more accurately in a golf putting task and displayed more effective visual gaze, putting kinematics, and muscle activity than participants who exhibited threat CV reactivity. Turner et al. (2012) found that challenge CV reactivity was related to better netball shooting performance from baseline levels, performed $24 \mathrm{hr}$ after CV data collection. In sum, challenge $\mathrm{CV}$ reactivity is associated with superior athletic performance.

The present study examined whether challenge and threat $\mathrm{CV}$ reactivity to a stressor (description of upcoming pressured batting) can predict subsequent performance of elite cricketers in a pressured batting test. This study contributes to the extant literature in two main ways. First, the current study uses a skilled population, elite academy cricketers (comprising the top-30 players in their age group in the UK and 12 players from a professional county cricket club) and builds on the work of Blascovich et al. (2004), who used high-level varsity athletes. Second, performance is determined by a specifically designed (by national cricket coaches) one-off pressured batting test, offering a more valid assessment of how CV reactivity relates to imminent and pressured performance than previous studies. Based on the TCTSA and previous research findings (e.g., Blascovich et al., 2004; Moore et al., 2012; Turner et al., 2012), it was hypothesized that $\mathrm{CV}$ reactivity indicating challenge would be associated with self-reported evaluations of challenge, higher selfefficacy, higher perceived control, a greater focus on approach goals, higher levels of positive emotions, and superior performance in the Batting Test, compared with $\mathrm{CV}$ reactivity indicating threat.

\section{Method}

\section{Participants}

Forty-two elite-level national $(n=30)$ and county $(n=$ $12)$ male academy cricketers $\left(M_{\text {age }}=16.45\right.$ years, $S D_{\text {age }}$ $=1.38$ years; $M_{\exp }=8.40$ years, $S D_{\exp }=2.44$ years). ${ }^{1}$ Participants represented two typical roles in cricket: batsmen $(n=25)$ and bowlers $(n=17)$. No inducement was offered to participants for taking part. All participants reported being normotensive and in good health. Ethical approval was granted from the university and individual informed consent was obtained before all data collection. 


\section{Measures}

Cardiovascular. A bio-impedance cardiograph integrated system (model HIC-3004), along with a blood pressure (BP) monitor (Suntech Tango+) was used to measure all cardiac and vascular responses, following guidelines presented by Sherwood (1993). Impedance cardiographic (ZKG) and electrocardiographic (ECG) recordings provided continuous measurement of $\mathrm{CV}$ performance. Impedance cardiograph measurement used a tetrapolar band electrode configuration widely used in similar research (see Blascovich et al., 2011). External ECG recordings were obtained using a Lead II configuration (right arm, left arm, and left leg). A Suntech Tango+ Stress Test BP monitor was used to record continuous noninvasive blood pressure from the brachial artery of the participant's nonpreferred arm. CopWin integrated the ZKG, ECG, and BP recordings to provide the four CV indices that differentiate challenge and threat. Specifically, HR, PEP, CO, and TPR were used.

Emotions. For brevity, emotions were assessed using five items in the Sport Emotion Questionnaire (SEQ; Jones, Lane, Bray, Uphill, \& Catlin, 2005). These were anger, anxiety, dejection, excitement, and happiness, and participants indicated how they felt about the imminent Batting Test on a 5-point Likert scale ranging from 0 (not at all) to 4 (extremely). In addition, a single item asked participants to indicate how helpful they perceived their emotional state to be on a 5-point Likert scale ranging from 0 (not at all) to 4 (extremely).

Achievement Goals. The Achievement Goals Questionnaire (AGQ; Conroy, Elliot, \& Hofer, 2003) measures mastery approach goals, mastery avoidance goals, performance approach goals, and performance avoidance goals. The AGQ was reduced to four items (one item for each subscale). Participants were asked how they felt about the imminent Batting Test on a 7-point Likert scale ranging from 1 (not at all true) to 7 (very true).

Self-Efficacy. Participants responded to two items, "to what extent do you feel confident that you can score highly" and "to what extent do you feel confident that you can make the right shot decisions/selections?" The participants responded on a 5-point Likert scale ranging from 1 (not at all) to 5 (completely).

Perceived Control. Adapted from the Academic Control Scale (Perry, Hladkyj, Pekrun, \& Pelletier, 2001), participants were asked to rate how much they agreed that "The more effort I put into this test, the better I will do" on a 5-point Likert scale ranging from 1 (strongly disagree) to 5 (strongly agree).

Cognitive Evaluation. Participants indicated how challenged or threatened they felt about the imminent Batting Test on a 9-point Likert scale ranging from -4 (threatened) to +4 (challenged).

Task Importance. Participants were asked how important doing well in the imminent Batting Test was for them on a 6-point Likert scale ranging from 0 (not at all) to 5 (very much so).

The Batting Test. The Batting Test is conducted periodically with all cricketers at national academy level and assesses a cricketer's ability to perform under pressured simulated match circumstances. The Batting Test took place at a top-level training facility on three separate occasions (two for national-level athletes and one for county-level athletes). Participants performed alone and were given 30 deliveries from a pace bowling machine (set at $80 \mathrm{mph}$ ) from which they were to score 36 runs in total. Runs were allocated by coaching staff who decided how many runs each shot was worth depending on the speed and trajectory of the ball in relation to the position of the field. As would be required in an actual cricket match, participants had to run the number of runs allocated by the coaching staff (unless a boundary was scored). The position of the field was formed using cones placed in the following positions: fine leg, deep square leg, midwicket, mid-on, third man, point, cover (sweeper), cover (saving one run), mid-off, wicket keeper, and bowler (field reversed for left-handed batsman). For each shot, participants could score zero, one, two, three, four, or six runs. Five runs were deducted for any dismissal (bowled, caught, or Leg Before Wicket), which was decided by the coaching staff, and the participants continued the test until 30 balls had been faced, even if the 36-run target had been reached.

Performance. Batting Test scores for each participant were calculated by adding all runs scored and subtracting five runs per dismissal.

\section{Procedures}

Laboratory Setup. Data collection took place in a laboratory at each academy training facility on three separate occasions. Participants were asked to refrain from participating in heavy exercise in the $24 \mathrm{hr}$ before data collection and to refrain from consuming caffeine, food, and sports drinks in the $2 \mathrm{hr}$ preceding their laboratory appointment. On entry to the laboratory, participants were given a brief outline of the CV data collection protocol to demystify the process and equipment.

Participant Preparation. Participants were prepared for data collection in the same manner as in Turner et al. (2012), and followed relevant guidelines (Blascovich et al., 2011; Sherwood, 1993). This included placement of band electrodes, spot electrodes, and BP cuff, and attachment to the impedance cardiogram. Participants were then informed that there would be a 5-min rest period, after which they would hear a set of audio-taped instructions.

Cardiovascular and Psychological Data Collection. Cardiovascular data recording continued through the 5-min rest period (baseline) and posttask instruction phases. Standardized audio-taped task instructions 
(available on request from the first author) lasted for $1 \mathrm{~min}$ and were designed to induce perceptions of a motivated performance situation as in previous studies (e.g., Turner et al., 2012). The instructions informed participants that the Batting Test assesses an ability to perform under pressure, that they would be required to face 30 balls and attain 36 runs to be successful, and that their total score would be compared with all other participants (ego threatening). The instructions also stated that coaches would consider their performance in the Batting Test when making future decisions about program selection, and therefore they would have to try very hard to perform well. Similar types of instructions have been successfully used in previous competitive settings as a stressor (e.g., Barker, Jones, \& Greenlees, 2010; Hardy, Beattie, \& Woodman, 2007). Participants were then asked to mentally prepare for the upcoming Batting Test for 2 min. After the 2 min of mental preparation, participants completed all self-report measures in relation to the imminent Batting Test.

Manipulation Check. At the end of the self-report measures, participants were asked to detail their thoughts in the 2 min of mental preparation time after hearing the instructions. This item was used to determine the extent participants engaged in task-relevant thoughts while $\mathrm{CV}$ data were being recorded. Participants responded by writing their thoughts on a designated answer sheet.

Batting Test Performance. After CV and self-report data collection, participants were informed that the test would begin in $30 \mathrm{~min}$, during which time they were to change into all necessary batting equipment. When the participants arrived at the nets, coaching staff reminded them of the specific rules (30 balls to get 36 runs, 5-run deductions for dismissals) and the field positions. Participants were given two "sighters" (practice deliveries) from the pace bowling machine to help familiarize them with the speed and pitch of the ball. The test began with the delivery of the next ball. After the Batting Test, participants received a full debrief.

\section{Analytic Strategy}

As in previous studies (Turner et al., 2012), ShapiroWilk tests were performed before main analyses. If the presence of outliers were indicated, then $z$ scores for significant outliers were assessed (e.g., Mendes, Reis, Seery, \& Blascovich, 2003). Data with $z$ scores greater than 2 were omitted from further analyses. The analytic strategy for the CV data comprised six steps. First, in line with previous studies using a similar protocol (e.g., Mendes et al., 2003; Turner et al., 2012), HR and PEP averaged across the 3 -min posttask ( 1 min of task instructions and 2 min of mental preparation) phase were compared with HR and PEP in the last minute of the baseline $\mathrm{CV}$ data collection phase. This was to determine whether the task represented a motivated performance situation for participants. Second, differences in CV reactivity and performance were explored between participant level (national academy, county academy) and role (batsmen, bowlers). Third, hierarchical multiple regression was used in two steps to predict Batting Test performance with TPR and CO reactivity. Participant cricket experience, level, and role, was entered in Step 1 , and either CO reactivity or TPR reactivity were entered into Step 2. Cardiovascular reactivity scores were calculated for $\mathrm{CO}$ and TPR by subtracting the raw $\mathrm{CV}$ responses for the last minute of baseline $\mathrm{CV}$ data collection phase from the average raw $\mathrm{CV}$ responses across the 3-min posttask instructions CV data collection phase (Seery, Weisbuch, \& Blascovich, 2009). Fourth, in line with similar research (e.g., Blascovich et al., 2004; Turner et al., 2012), average CO and TPR reactivity were combined into a single challenge and threat index, and a single hierarchical multiple regression analysis was conducted to predict Batting Test performance with the challenge and threat index. Fifth, individual differences in the $\mathrm{CV}$ reactivity-batting performance relationship were explored by conducting independent $t$ tests examining differences in the self-report variables between participants who performed well in a challenge state with those who did not, and differences in the self-report variables between participants who performed poorly in a threat state with those who did not. Finally, Pearson's correlation analyses were conducted to examine the association between CV reactivity, self-reported psychological states, and performance. All multicollinearity, normality, and outlier checks met the assumptions necessary for all data analyses.

\section{Results}

\section{Task Engagement}

Two separate paired-samples $t$ tests were conducted to compare the last minute of baseline HR and PEP with HR and PEP averaged across the 3 -min posttask instruction $\mathrm{CV}$ data collection phase for all participants. ${ }^{2}$ For HR, there was a significant increase, $t(38)=3.97, p<.001$, from the last minute $(M=72.54 \mathrm{bpm}, S D=10.28 \mathrm{bpm})$ of baseline to the posttask instruction phase $(M=74.50$ $\mathrm{bpm}, S D=10.12 \mathrm{bpm})$. For PEP, there was a significant attenuation, $t(41)=4.09, p<.001$, from the last minute $(M=133.10 \mathrm{~ms}, S D=18.53 \mathrm{~ms})$ of baseline to the posttask instruction phase $(M=130.46 \mathrm{~ms}, S D=18.13 \mathrm{~ms})$. Heart rate and PEP reactivity indicated that participants engaged in the task, an important prerequisite for the analysis of challenge and threat $\mathrm{CV}$ reactivity (Blascovich et al., 2011). In addition, participants indicated that Batting Test success was important to them $(M=4.30, S D=$ $.72), t(39)=37.60, p<.001$, and the manipulation check indicated that all participants engaged in task-relevant thoughts while thinking about the upcoming Batting Test, supporting CV data suggesting that participants engaged in the task. ${ }^{3}$ 


\section{Differences Between Participants' Levels and Roles}

Independent $t$ tests revealed that there were no significant differences between county academy participants and national academy participants in TPR reactivity, $t(40)=$ $.29, p>.05$; CO reactivity, $t(40)=.14, p>.05$; or performance, $t(40)=.17, p>.05$. In addition, there were no significant differences between batsmen and bowlers in TPR reactivity, $t(40)=.68, p>.05 ;$ CO reactivity, $t(40)$ $=1.27, p>.05$; or performance, $t(40)=.46, p>.05$.

\section{Cardiovascular Reactivity and Performance}

To examine the relationships between TPR and CO reactivity and performance, two separate hierarchical multiple regression analyses were carried out, with total Batting Test score as the outcome variable (controlling for cricket experience, level, and role) predicted by either $\mathrm{CO}$ or TPR reactivity. Level and role were included in Step 1 to account for the potential influence of these variables in predicting performance in the regression analyses. Cricket experience, level, and role, were entered in Step 1; in Step 2, either CO reactivity or TPR reactivity was entered. In Step 1, a significant proportion of variance was not accounted for, $R^{2}=.05, p>.05$. For TPR, a significant proportion of variance was accounted for by the addition of Step $2, R^{2}=.26, p=.001$. Higher TPR was significantly associated with lower total score $(b=-.10, \beta=-.51)$. For $\mathrm{CO}$, a significant proportion of variance was accounted for by the addition of Step $2, R^{2}=.31, p<.001$. Higher $\mathrm{CO}$ was significantly associated with higher total score $(b=27.59, \beta=.57)$.
Challenge and Threat Index and Performance. A single challenge and threat index was calculated by converting average $\mathrm{CO}$ and average TPR reactivity values into $z$ scores and summing them. Cardiac output was assigned a weight of +1 whereas TPR was assigned a weight of -1 , so that larger values reflected challenge reactivity. Following previous research (e.g., Blascovich et al., 2004), the index allows the pattern of reactivity to be assessed in one hierarchical multiple regression analysis, and accounts for the interrelatedness of $\mathrm{CO}$ and TPR reactivity measures. To examine the relationships between the challenge and threat index and performance, a hierarchical multiple regression analysis was carried out, with performance as the outcome variable, controlling for cricket experience, level, and role, predicted by the challenge and threat index. Cricket experience, level, and role, were entered in Step 1, and in Step 2 the challenge and threat index was entered. In Step 1 a significant proportion of variance was not accounted for, $R^{2}=.07, p$ $>$.05. The addition of the challenge and threat index in Step 2 made a significant contribution to the proportion of variance accounted for in the model, $R^{2}=.41, p<.001$. A higher challenge and threat index value (indicating challenge reactivity) was significantly associated with higher total score $(b=4.61, \beta=.65)$.

Challenge and Threat Index, Psychological Components of the TCTSA, and Performance. Pearson's correlation analyses ${ }^{4,5}$ (Table 1) revealed significant positive associations $(p<.05)$ between the challenge and threat index and helpfulness of emotional state $(r=.36)$. In addition, performance was positively and significantly associated with performance approach goals $(r=.35)$

Table 1 Mean $\pm S D$ and Correlation Analyses for Performance, Psychological Variables, and the Challenge and Threat Index

\begin{tabular}{lccc}
\hline Variable & $\boldsymbol{M} \pm \mathbf{S D}$ & $\begin{array}{c}\text { Challenge and } \\
\text { Threat Index }\end{array}$ & Performance \\
\hline Performance & $27.09 \pm 11.80$ & $.64^{* *}$ & - \\
Anxiety & $1.93 \pm 1.11$ & -.07 & -.09 \\
Excitement & $2.62 \pm .88$ & -.04 & -.18 \\
Happiness & $2.28 \pm .75$ & .03 & -.19 \\
Helpfulness of Emotional State & $3.24 \pm .73$ & $.36^{*}$ & .17 \\
Performance Approach Goals & $6.63 \pm .63$ & .25 & $.35^{*}$ \\
Performance Avoidance Goals & $3.38 \pm 1.35$ & -.09 & -.14 \\
Mastery Approach Goals & $5.20 \pm 1.40$ & -.10 & -.06 \\
Mastery Avoidance Goals & $2.98 \pm 1.44$ & -.07 & -.01 \\
Control & $4.10 \pm 1.08$ & .14 & .10 \\
Self-Efficacy & $3.84 \pm .67$ & .30 & $.33^{*}$ \\
Cognitive Evaluations & $2.31 \pm 1.08$ & .21 & .02 \\
Task Importance & $4.30 \pm .72$ & -.01 & -.19 \\
\hline
\end{tabular}

$* p<.05 ; * * p<.01$. 
and self-efficacy $(r=.33)$. All other correlations were nonsignificant and the effect sizes associated with the correlations were small to medium (Cohen, 1992).

\section{Exploring Individual Differences in the CV Reactivity-Batting Test Performance Relationship}

While in general challenge $\mathrm{CV}$ reactivity predicts success and threat $\mathrm{CV}$ reactivity predicts poor performance, this relationship did not hold for all participants. We explored the role that psychological aspects may play in explaining these individual differences.

Threat CV Reactivity and Batting Test Success. Five participants exhibited threat $\mathrm{CV}$ reactivity but reached or almost reached (within 2 standard errors of mean performance) the Batting Test target score of 36 runs. Self-report scores for the 5 participants were compared with the 17 participants who exhibited threat $\mathrm{CV}$ reactivity but performed poorly. Independent $t$ tests revealed a significant difference in self-efficacy, $t(20)=.42, p<$ .02 , between the 5 participants $(M=4.50, S D=.50)$ who performed well and the 17 participants who performed poorly $(M=3.62, S D=.65)$. All means, standard deviations, and nonsignificant differences can be seen in Table 2.

Challenge CV Reactivity and Batting Test Failure. Six participants exhibited challenge CV reactivity but did not reach or almost reach (within 2 standard errors of mean performance) the Batting Test target score of 36 runs. Self-report scores for the 6 participants were compared with the 14 participants who exhibited challenge $\mathrm{CV}$ reactivity and did reach or almost reach the runs target. Independent $t$ tests revealed a significant difference in performance avoidance goals, $t(17)=2.25, p<$ .03 , between the 6 participants who performed poorly $(M=4.50, S D=1.52)$ and the 14 participants who performed well $(M=2.92, S D=1.19)$. All means, standard deviations, and nonsignificant differences can be seen in Table 2.

\section{Discussion}

The current study is the first to demonstrate that imminent performance in elite athletes can be predicted by challenge and threat $\mathrm{CV}$ reactivity. Specifically, in response to a psychological stressor (description of the Batting Test), challenge $\mathrm{CV}$ reactivity predicted superior performance in the Batting Test, compared with threat $\mathrm{CV}$ reactivity. However, overall, the associations between CV reactivity and self-report responses were weak. It was also found that the small number of participants who exhibited threat reactivity but performed well had significantly higher levels of self-efficacy than participants who exhibited threat reactivity and performed poorly. In addition, a small number of participants exhibited challenge CV reactivity but performed poorly. When compared with participants who exhibited challenge reactivity and performed well, participants who performed poorly had significantly higher performance avoidance goals. Therefore, differences in psychological approach may in part explain the small number of instances of countertheoretical performance effects.

The present paper supports growing research showing that $\mathrm{CV}$ indicators of challenge and threat states relate to athletic performance. This study also supports theoretical predictions (BPS model and TCTSA) that CV indicators of a challenge state should be associated with superior performance in motivated performance situations. Importantly, the current paper extends research by using an elite athlete sample in an imminent and more personally relevant performance setting than used in previous research (e.g., Moore et al., 2012; Turner et al., 2012). Notably, CV reactivity was able to predict performance $30 \mathrm{~min}$ after $\mathrm{CV}$ data collection. This finding adds to research showing that $\mathrm{CV}$ reactivity to a stressor (description of an upcoming task) predicts performance in both immediate (Moore et al., 2012) and more delayed competitions (Blascovich et al., 2004; Turner et al., 2012).

The relationship between CV reactivity and performance in the current study, which was the main focus, was consistent and strong. Cardiovascular reactivity measured the participants' immediate reactions to being told about the upcoming Batting Test, which is a scenario analogous to situations in which an athlete receives information about an important competition. For example, a cricketer next in the batting order seeing his teammate get dismissed and realizing he is next to bat has to prepare mentally to execute skills and tactics relevant to the specific match situation. In other words, CV reactivity in the minutes following the onset of a psychological stressor is an important indicator of how athletes will subsequently perform. Exactly how CV reactivity exerts an influence on imminent and delayed performance is not evidenced in the current study, but some mechanisms can be postulated.

It is possible that challenge $\mathrm{CV}$ reactivity exhibited in response to the stressor (description of the Batting Test) reflected more helpful thoughts and feelings on approach to the motivated performance situation (e.g., Chalabaev et al., 2009), although self-report data from previous research (e.g., Turner et al., 2012) and the current study do not fully support this assertion. Alternatively, physiological factors could help to explain the performance effects, particularly if the responses to the description of the competition are mimicked in the event itself. For example, muscular tension is likely to be higher in a threat state than in a challenge state (Wright \& Kirby, 2003), which has obvious implications for skilled motor performance. Further, Moore et al. (2012) found that compared with a threat state, a challenge state was marked by more effective movement patterns (kinematics) and less muscular activation, widely recognized as contributing to successful motor performance (e.g., Lay, Sparrow, Hughes, \& O'Dwyer, 2002). Based on recent findings, a full investigation on the potential psychophysiological mechanisms causing observed performance effects in challenge and threat states is warranted. 


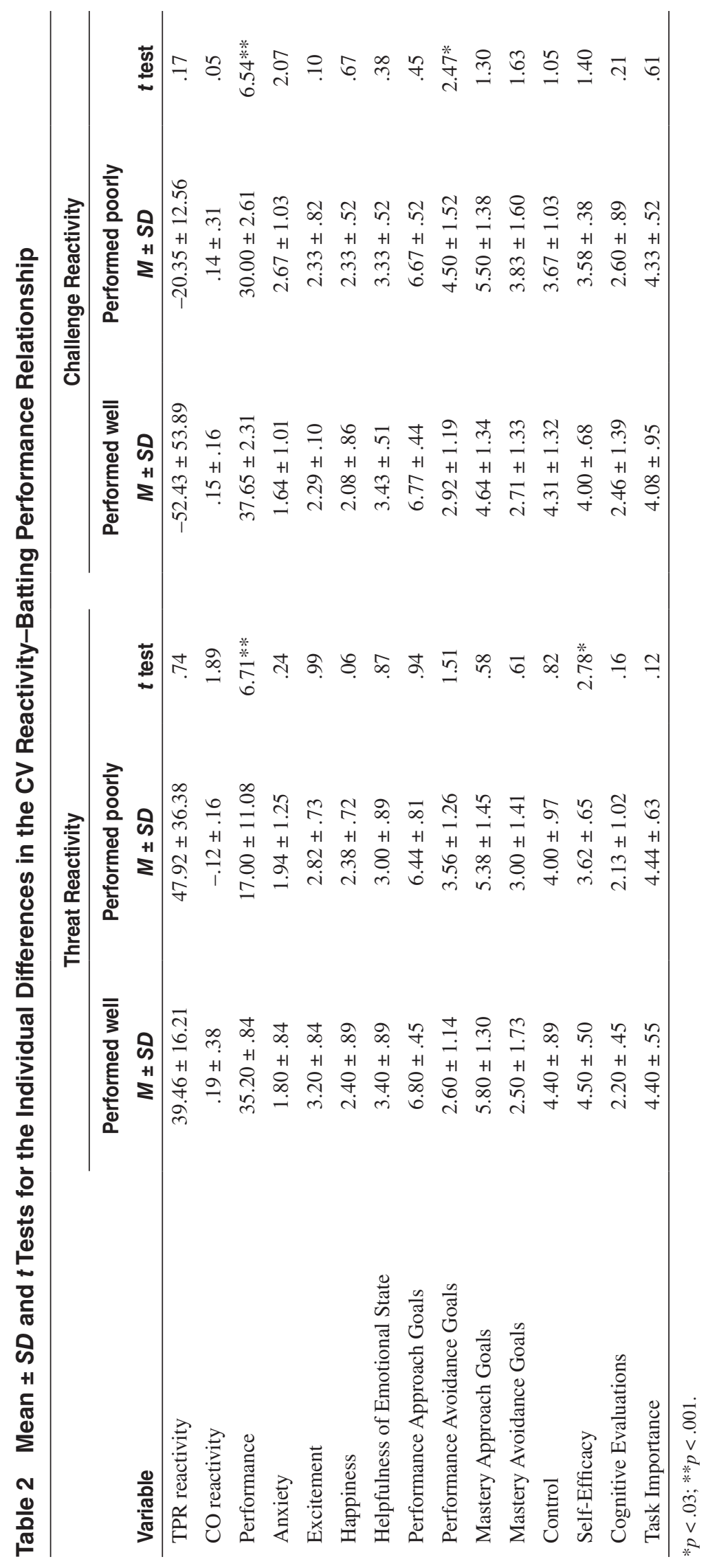


In the current study, relationships between $\mathrm{CV}$ reactivity and self-reported psychological states were weak and inconsistent. The only significant relationship to emerge was a positive association between the challenge and threat index and helpfulness of emotional states. To explain, higher challenge $\mathrm{CV}$ reactivity was related to more helpful perceptions of emotional states before the Batting Test. Although this finding is in the hypothesized direction, the findings were largely unsupportive of the TCTSA's predictions concerning cognitive and emotional correlates of challenge and threat states. Research testing the predictions of the TCTSA with regard to the relationship between psychological states and $\mathrm{CV}$ reactivity is beginning to emerge (e.g., Williams \& Cumming, 2012; Turner et al., 2012) and this growing body of evidence should help to elucidate the relationship between psychological states and CV reactivity, and help to refine the TCTSA.

There were also significant positive associations between Batting Test performance and self-reported performance approach goals and self-efficacy. Specifically, a higher focus on approach goals and higher self-efficacy was related to superior performance in the Batting Test. These findings are consistent with the predictions of the TCTSA, and support much of the sport psychology literature. For example, the positive link between selfefficacy and sports performance is widely recognized (e.g., Bandura, 2006). The positive association between performance approach goals and performance found in the current paper is also consistent with theory and research (Chalabaev et al., 2009; Jones et al., 2009) and is especially plausible considering the nature of the Batting Test. To explain, performance approach goals reflect a motivation to be seen as more competent that other persons, and with comparison between participants emphasized in the Batting Test instructions before performance, it is perhaps unsurprising that a higher focus on performance approach goals was related to better performance. It should be noted that in a cricket academy context, even though the cricketers perform together as a team, comparison between cricketers is ubiquitous and necessary (Barker et al., 2011) to facilitate future academy selection decisions, perhaps reflected by the performance approach goals results in the current paper.

An intriguing result in the current study was that some participants exhibited threat reactivity but performed well, and some participants exhibited a challenge state but performed poorly, representing an important exception to the predictive reliability of challenge and threat $\mathrm{CV}$ reactivity. These findings are interesting because they suggest that some individuals, despite exhibiting a challenge or threat state, performed contrary to our expectations and theoretical predictions. Investigation of self-report responses showed that participants who performed well after exhibiting threat $\mathrm{CV}$ reactivity had higher self-efficacy, whereas those who performed poorly after exhibiting challenge $\mathrm{CV}$ reactivity had higher avoidance goals. Suggesting cognitions do have a role to play in either inoculating against the influence of threat CV reactivity on skilled performance, or marring the effect of challenge reactivity on performance. This finding is consistent with previous research outlining the interplay between psychological states and emotional responses such as Jones's (1995) model of debilitative and facilitative competitive state anxiety and Hardy's butterfly catastrophe model (Hardy, 1990). It is also consistent with previous challenge and threat research (Hoyt $\&$ Blascovich, 2010) that suggests that individuals who exhibit threat $\mathrm{CV}$ reactivity but report high self-efficacy may be reacting to the threat of the situation in a way that allows maintained or improved performance. Although interesting, the findings that suggest cognitions may moderate the $\mathrm{CV}$ reactivity-performance relationship should be considered cautiously, as the analysis lacked statistical power (5 vs. 17 in the threat "group," and 6 vs. 14 in the challenge "group"). Future research could aim for sufficient participant numbers to statistically test for the moderation effects of resource appraisals on the $\mathrm{CV}$ reactivity-performance relationship. ${ }^{6}$

Despite some significant relationships and interesting findings emerging for self-report data in the current study, the relationships between self-reported psychological states and CV reactivity were weak and inconsistent. These absent associations could be explained in various ways. First, the social desirability inherent in elite sport settings may cause elite athletes to respond in a biased way to questions concerning psychological states (Williams \& Krane, 1992). Second, many of the self-reported psychological constructs were measured using shortened versions of the measures they derived from to shorten the time between self-report data collection and batting test performance. For example, only one item (as opposed to four) assessed each type of achievement goal, and the SEQ measuring emotions was reduced from 22 items to 5 items, 1 for each subscale. This shortening may have altered scale reliability and limited participant responses to one opportunity to express their psychological experiences. Third, many authors suggest that self-reports are a poor window into how individuals process consciously available evaluations, and no window at all into processes that may occur unconsciously, such as the immediate evaluation of a stressor (e.g., LeDoux, 1998; Blascovich $\&$ Mendes, 2000). There is evidence that the subconscious awareness of evocative stimuli, thus bypassing measurable cognitive evaluation, can determine $\mathrm{CV}$ responses (e.g., Weisbuch-Remington, Mendes, Seery, \& Blascovich, 2005), and numerous challenge and threat studies illustrate a disjunction of $\mathrm{CV}$ responses with those that are under conscious control (e.g., self-report; Mendes, Blascovich, Lickel, \& Hunter, 2002). Finally, challenge and threat states may be more difficult to assess via self-report measures than through $\mathrm{CV}$ reactivity (Chalabaev et al., 2009). Therefore, it is still important that techniques that do not depend on verbal reports are used (Scherer, 1993), or that questionnaires be less susceptible to response bias, or be more able to assess deeper cognitions. For instance, perhaps a measure of dispositional evaluations (e.g., Roesch \& Rowley, 2005) is necessary, alongside 
specifically validated measures that assess challenge and threat states in athletes.

There are some limitations to the current study, which outline potential areas of future research. Unlike the recent study by Turner et al. (2012), we were unable to measure baseline batting performance. However, the participants were selected because of their elite athletic status and it was possible to statistically control for differences in cricket experience and level as recommended by previous research (e.g., Derks, Scheepers, van Laar, $\&$ Ellemers, 2011). That said, the findings in the present paper that batsman did not perform better in the pressured Batting Test than bowlers and that national and county academy players did not differ are intriguing. It may suggest that the Batting Test, despite its frequent use at academy level, is not a valid performance measure, although it could also reflect that the Batting Test presents participants with a specific scenario and one that lower order batsmen (who may have a primary bowling role within the team) may be more familiar with than, for example, the opening batsmen. Future research could obtain baseline performance in an elite athletic sample so that changes in performance from the norm can be ascertained, and to explore the validity and reliability of the Batting Test as a performance measure. In addition, the Batting Test was rendered meaningful for the athletes by describing the evaluative circumstances of the test, and by creating a pseudo-match scenario. But actual competitive performance is likely to be more meaningful to athletes, and therefore potentially more stressful, which may influence $\mathrm{CV}$ reactivity and change its relationship to psychological and performance variables. While the performance context adequately elicited $\mathrm{CV}$ reactivity indicating engagement (increased HR and decreased PEP; Blascovich et al., 2011), the mean changes in HR were small compared with other challenge and threat research studies (e.g., Blascovich et al., 2004). Alternatively, the knowledge of a 30-min gap between stressor and performance may have had a diminishing effect on HR that might not occur if $\mathrm{CV}$ data were collected directly before performance (e.g., Epstein \& Fenz, 1962; Moore et al., 2012). Finally, it may be suggested that a better method to explore challenge and threat states and performance would be to create challenge and threat conditions similar to previous research (e.g., Moore et al., 2012; Tomaka, Blascovich, Kibler, \& Ernst, 1997) and examine the between-conditions performance effects. However, in competition, individuals are not usually artificially oriented to challenge or threat states, but appraise the situation quickly and often unconsciously (e.g., LeDoux, 1998). Therefore, we felt that inducing psychological stress in the participants and then exploring their unconditioned responses was more realistic and ecologically valid.

The findings of the current study have implications for the assessment and development of elite athletes. Cardiovascular reactivity measured $30 \mathrm{~min}$ before an important competition predicted athletic performance, offering clear applications to sport settings, especially for sport psychology practitioners. This type of assessment eliminates the social desirability inherent in self-report measures. Alongside other psychological and behavioral screening tools, a psychologist can form a detailed picture of when athletes will flourish, or succumb under pressure. Further, by measuring an athlete's $\mathrm{CV}$ reactivity in relation to their thoughts about an upcoming competition, it is possible to determine how the athlete will approach that situation, prompting interventions to promote a challenge state. For example, research has shown that individuals encouraged via instructional sets to think of themselves "capable of meeting that challenge" (p. 72) approach tasks as a challenge and exhibit challenge CV reactivity (Tomaka et al., 1997). In addition, athletes undertaking challenge imagery appraise upcoming tasks as a challenge, experience facilitative anxiety, and feel more in control (e.g., Hale \& Whitehouse, 1998; Williams \& Cumming, 2012; Williams, Cumming, \& Balanos, 2010). Specifically, in one study (Williams et al., 2010) a challenge imagery script emphasized that the athlete's resources met the demands of the situation, that they could be confident (high self-efficacy), demonstrate competence (high perceived control), and had a lot to gain (approach goals). Results showed that the challenge script led athletes to feel that their emotional response was more helpful for performance, were more confident, and appraised the situation as less threatening.

For athlete development, $\mathrm{CV}$ reactivity information could help athletes to better understand their responses to pressure, thus encouraging them to seek assistance and guidance in strategies to enhance their ability to deal with pressure. It is also possible to assess the effects of stress inoculation on athletes by repeatedly exposing them to pressure situations such as the Batting Test and recording $\mathrm{CV}$ reactivity before their performance. In fact, research has shown that prior task exposure (Kelsey, Blascovich, Tomaka, Leitten, Schneider, \& Wiens, 1999) and stress inoculation programs integrating visualization, self-talk, and relaxation strategies (Mace \& Carroll, 1989) can diminish the effects of sympathetic adreno-medullary activity on the heart, which may render the athletes more relaxed for performance under pressure. Indeed, prior task exposure may have had an influence on the results of the current study and future research should obtain prior exposure information to help statistically control for it in analyses.

To conclude, this is the first study to show that challenge and threat $\mathrm{CV}$ reactivity can predict imminent sport performance in elite athletes. Specifically, challenge CV reactivity predicted superior performance in a pressured cricket Batting Test compared with threat CV reactivity. It was also found that participants who exhibited threat $\mathrm{CV}$ reactivity but performed well reported greater self-efficacy than participants who exhibited threat CV reactivity but performed poorly. In addition, participants who exhibited challenge $\mathrm{CV}$ reactivity but performed poorly reported higher avoidance goals than participants who exhibited challenge $\mathrm{CV}$ reactivity and performed well. Sport psychologists could explore strategies to 
promote a challenge state, given the evidence beginning to emerge attesting to the benefits of a challenge state for performance. The present paper suggests that the assessment of $\mathrm{CV}$ reactivity may be a valid way of predicting pressured sport performance in elite athletes, and as such could be used to help form a more complete picture of how able an athlete is to reach their potential in motivated-performance situations.

\section{Notes}

1. A further five participants' data were collected but omitted due to poor quality impedance signal $(n=3)$ and failure to attend that Batting Test $(n=2)$.

2. Data for HR from three participants were omitted as they were outliers.

3. Data for task importance from two participants were omitted as they were outliers.

4. Participants data for happiness $(n=2)$, helpfulness of emotion $(n=1)$, performance approach goals $(n=2)$, performance avoidance goals $(n=2)$, mastery approach goals $(n=1)$, mastery avoidance goals $(n=1)$, control $(n=2)$, self-efficacy $(n=1)$, cognitive evaluation $(n=3)$, and task importance $(n=2)$ were omitted as they were outliers.

5. Variables Anger $(M=.00, S D=.00)$ and Dejected $(M=$ $.18, S D=.38$ ) were omitted from all analysis because of their low total scores.

6. We conducted a series of regression analyses to examine potential moderation effects of the resource evaluations on the $\mathrm{CV}$ reactivity-performance relationship with the sample as a whole, and for the challenge and threat "groups" separately. No significant moderation effects emerged. These are available from the first author.

\section{References}

Bandura, A. (2006). Guide for constructing self-efficacy scales. In F. Pajares \& T. Urdan (Eds.), Self-efficacy beliefs of adolescents (Vol. 5, pp. 307-337). Greenwich, CT: Information Age Publishing.

Barker, J.B., Jones, M.V., \& Greenlees, I. (2010). Assessing the immediate and maintained effects of hypnosis on self-efficacy and soccer wall-volley performance. Journal of Sport \& Exercise Psychology, 32, 243-252. PubMed

Barker, J.B., McCarthy, P.J., \& Harwood, C.G. (2011). Reflections on consulting in elite youth male English cricket and soccer academies. Sport and Exercise Psychology Review, 7, 58-72.

Blascovich, J., \& Mendes, W.B. (2000). Challenge and threat appraisals: The role of affective cues. In J.P. Forgas (Ed.), Feeling and thinking: The role of affect in social cognition (pp. 59-82). Paris: Cambridge University Press.

Blascovich, J., Mendes, W.B., Vanman, E., \& Dickerson, S. (2011). Social Psychophysiology for Social and Personality Psychology. London: Sage.

Blascovich, J., Seery, M.D., Mugridge, C.A., Norris, K., \& Weisbuch, M. (2004). Predicting athletic performance from cardiovascular indexes of challenge and threat. Journal of Experimental Social Psychology, 40, 683-688. doi:10.1016/j.jesp.2003.10.007

Chalabaev, A., Major, B., Cury, F., \& Sarrazin, P. (2009). Physiological markers of challenge and threat mediate the effects of performance-based goals on performance. Journal of Experimental Social Psychology, 45, 991-994. doi:10.1016/j.jesp.2009.04.009

Cohen, J. (1992). A power primer. Psychological Bulletin, 112, 155-159. PubMed doi:10.1037/0033-2909.112.1.155

Conroy, D.E., Elliot, A.J., \& Hofer, S.M. (2003). A 2 x 2 achievement goals questionnaire for sport: Evidence for factorial invariance, temporal stability, and external validity. Journal of Sport \& Exercise Psychology, 25(4), 456-476.

Derks, B., Scheepers, D., Van Laar, C., \& Ellemers, N. (2011). The threat vs. challenge of car parking for women: How self- and group affirmation affect cardiovascular responses. Journal of Experimental Social Psychology, 47, 178-183. doi:10.1016/j.jesp.2010.08.016

Dienstbier, R.A. (1989). Arousal and physiological toughness: Implications for mental and physical health. Psychological Review, 96, 84-100. PubMed doi:10.1037/0033295X.96.1.84

Dienstbier, R.A. (1992). Mutual impacts of toughening on crises and losses. Faculty Publications: Department of Psychology, University of Nebraska-Lincoln.

Epstein, S., \& Fenz, W.D. (1962). Theory and experiment on the measurement of approach-avoidance conflict. Journal of Abnormal and Social Psychology, 64, 97-112. doi:10.1037/h0046187. PubMed

Hale, B.D., \& Whitehouse, A. (1998). The effects of imagery manipulated appraisal on intensity and direction of competitive anxiety. The Sport Psychologist, 12, 40-51.

Hardy, L. (1990). A catastrophe model of performance in sport. In G. Jones \& L. Hardy (Eds.), Stress and performance in sport (pp. 81-106). Chichester, England: Wiley.

Hardy, L., Beattie, S., \& Woodman, T. (2007). Anxiety-induced performance catastrophes: Investigating effort required as an asymmetry factor. The British Journal of Psychology, 98, 15-31. PubMed doi:10.1348/000712606X103428

Harrison, L.K., Denning, S., Easton, H.L., Hall, J.C., Burns, V.E., Ring, C., \& Carroll, D. (2001). The effects of competition and competitiveness on cardiovascular activity. Psychophysiology, 38, 601-606. PubMed doi:10.1111/14698986.3840601

Hoyt, C., \& Blascovich, J. (2010). The role of self-efficacy and stereotype activation on cardiovascular, behavioral and self-report responses in the leadership domain. The Leadership Quarterly, 21, 89-103. doi:10.1016/j. leaqua.2009.10.007

Jamieson, J.P., Koslov, K., Nock, M.K., \& Mendes, W.B. (2013). Experiencing discrimination increases risktaking. Psychological Science, 24(2), 131-139. PubMed doi: $10.1177 / 0956797612448194$

Jones, G. (1995). More than just a game: Research developments and issues in competitive anxiety in sport. The British Journal of Psychology, 86, 449-478. PubMed doi:10.1111/j.2044-8295.1995.tb02565.x 
Jones, M.V., Lane, A.M., Bray, S.R., Uphill, M., \& Catlin, J. (2005). Development and validation of the Sport Emotions Questionnaire. Journal of Sport \& Exercise Psychology, 27, 407-431.

Jones, M., Meijen, C., McCarthy, P.J., \& Sheffield, D. (2009). A theory of challenge and threat states in athletes. International Review of Sport and Exercise Psychology, 2, 161-180. doi:10.1080/17509840902829331

Jordet, G. (2009). Why do English players fail in soccer penalty shootouts? A study of team status, self-regulation, and choking under pressure. Journal of Sports Sciences, 27, 97-106. PubMed doi:10.1080/02640410802509144

Kelsey, R.M., Blascovich, J., Tomaka, J., Leitten, C.L., Schneider, T.R., \& Wiens, S. (1999). Cardiovascular reactivity and adaptation to recurrent psychological stress: Effects of prior task exposure. Psychophysiology, 36, 818-831. PubMed doi:10.1111/1469-8986.3660818

Lay, B.S., Sparrow, W.A., Hughes, K.M., \& O'Dwyer, N.J. (2002). Practice effects on coordination and control, metabolic energy expenditure, and muscle activation. Human Movement Science, 21, 807-830. PubMed doi:10.1016/ S0167-9457(02)00166-5

Lazarus, R.S., \& Folkman, S. (1984). Stress, appraisal, and coping. New York: Springer.

LeDoux, J. (1998). The emotional brain. London: Phoenix.

Mace, R.D., \& Carroll, D. (1989). The effect of stress inoculation training on self-reported stress, observer's rating of stress, heart rate and gymnastics performance. Journal of Sports Sciences, 7, 257-266. PubMed doi:10.1080/02640418908729846

Martens, R., Burton, D., Vealey, R.S., Bump, L.A., \& Smith, D.E. (1990). Development and validation of the Competitive State Anxiety Inventory-2. In R. Martens, R.S. Vealey, \& D. Burton (Eds.), Competitive anxiety in sport (pp. 117-190). Champaign, IL: Human Kinetics.

Mendes, W.B., Blascovich, J., Lickel, B., \& Hunter, S. (2002). Challenge and threat during social interactions with white and black men. Personality and Social Psychology Bulletin, 28, 939-952. doi:10.1177/01461672020280070.

Mendes, W.B., Reis, H.T., Seery, M.D., \& Blascovich, J. (2003). Cardiovascular correlates of emotional expression and suppression: Do content and gender context matter? Journal of Personality and Social Psychology, 84, 771-792. PubMed doi:10.1037/0022-3514.84.4.771

Moore, L.J., Vine, S.J., Wilson, M.R., \& Freeman, P. (2012). The effect of challenge and threat states on performance: An examination of potential mechanisms. Psychophysiology, 49(10), 1417-1425. PubMed doi:10.1111/j.14698986.2012.01449.x

Perry, R.P., Hladkyj, S., Pekrun, R.H., \& Pelletier, S.T. (2001). Academic control and action control in the achievement of college students: A longitudinal field study. Journal of Educational Psychology, 93(4), 776-789. doi:10.1037/0022-0663.93.4.776

Quested, E., Bosch, J.A., Burns, V.E., Cumming, J., Ntoumanis, N., \& Duda, J.L. (2011). Basic psychological needs satisfaction, stress-related appraisals, and dancers' cortisol and anxiety responses. Journal of Sport \& Exercise Psychology, 33, 828-846. PubMed
Roesch, S.C., \& Rowley, A.A. (2005). Evaluating and developing a multidimensional, dispositional measure of appraisal. Journal of Personality Assessment, 85, 188-196. PubMed doi:10.1207/s15327752jpa8502_11

Salvador, A. (2005). Coping with competitive situations in humans. Neuroscience and Biobehavioral Reviews, 29, 195-205. PubMed doi:10.1016/j.neubiorev.2004.07.004

Scherer, K.R. (1993). Studying the Emotion-Antecedent Appraisal Process. Cognition and Emotion, 7(3-4), 325-355. doi:10.1080/02699939308409192

Seery, M.D. (2011). Challenge or threat? Cardiovascular indexes of resilience and vulnerability to potential stress in humans. Neuroscience and Biobehavioral Reviews, 35, 1603-1610. PubMed doi:10.1016/j.neubiorev.2011.03.003

Seery, M.D., Weisbuch, M., \& Blascovich, J. (2009). Something to gain, something to lose: The cardiovascular consequences of outcome framing. International Journal of Psychophysiology, 73, 308-312. PubMed doi:10.1016/j. ijpsycho.2009.05.006

Sherwood, A. (1993). Use of impedance cardiography in cardiovascular reactivity research. In J. Blascovich \& E.S. Katkin (Eds.), Cardiovascular Reactivity to Psychological Stress and Disease (pp. 157-199). Washington, DC: American Psychological Association.

Tomaka, J., Blascovich, J., Kibler, J., \& Ernst, J.M. (1997). Cognitive and physiological antecedents of threat and challenge appraisal. Journal of Personality and Social Psychology, 73(1), 63-72. PubMed doi:10.1037/00223514.73.1.63

Turner, M.J., Jones, M.V., Sheffield, D., \& Cross, S.L. (2012). Cardiovascular indices of challenge and threat states predict performance under stress in cognitive and motor tasks. International Journal of Psychophysiology, 86(1), 48-57. PubMed doi:10.1016/j.ijpsycho.2012.08.004

Weisbuch-Remington, M., Mendes, W.B., Seery, M.D., \& Blascovich, J. (2005). The nonconscious influence of religious symbols in motivated performance situations. Personality and Social Psychology Bulletin, 31, 1203-1216. PubMed doi:10.1177/0146167205274448

Williams, S.E., \& Cumming, J. (2012). Challenge vs. threat: Investigating the effect of using imagery to manipulate stress appraisal of a dart throwing task. Sport \& Exercise Psychology Review, 8(1), 4-21.

Williams, S.E., Cumming, J., \& Balanos, G. (2010). The use of imagery to manipulate challenge and threat appraisal states in athletes. Journal of Sport \& Exercise Psychology, 32, 339-358. PubMed

Williams, J.M., \& Krane, V. (1992). Coping styles and selfreported measures of state anxiety and self-confidence. Journal of Applied Sport Psychology, 4(2), 134-143. doi:10.1080/10413209208406457

Wright, R.A., \& Kirby, L.D. (2003). Cardiovascular correlates of challenge and threat appraisals: A critical examination of the biopsychosocial analysis. Personality and Social Psychology Review, 7, 216-233. PubMed doi:10.1207/ S15327957PSPR0703_02

Manuscript submitted: November 17, 2012

Revision accepted: April 14, 2013 